\title{
Routing for Mobile UWSN: A Survey
}

\author{
${ }^{1}$ B.Priyalakshmi, K.Mahalakshmi ${ }^{2}$ \\ ${ }^{1}$ Research Scholar, Anna University, Chennai \\ ${ }^{2}$ Professor. Dept of IT, Karpagam College of Engineering, Coimbatore \\ *Corresponding Author Email: ${ }^{2}$ prof.dr.mlk@gmail.com
}

\begin{abstract}
Recently Underwater Wireless Sensor Networks (UWSNs) is now emerging as a important research area to explore underwater environments and its resource. Underwater wireless senor nodes are utilized for extensive variety of uses, for example, checking the marine condition for scientific to commercial investigation and coastline surveillance to underwater contamination observing, from water-based calamity preventions to water-based games facilitation. Due to the unstable underwater environment there is a need to increase the scalability and reduce the energy consumption. Connectivity and Coverage are the important properties that decide the proper detection and communication in UWSN. Many researchers have introduced the routing protocol which deals with mobility of node but the research investigation needs further advancement to model a powerful routing algorithm which control movement of the node. In this paper the literature survey on different routing protocol for mobile UWSN are discussed.
\end{abstract}

Keywords: WSN (wireless Sensor Network), UWSN (Underwater wireless sensor Network), UW sink, AUV, Routing, Energy efficiency.

\section{Introduction}

UWSN are emerging despite challenging applications such as, underwater surrounding observation, examination of coastal areas, pollution monitoring, and extraction of rare remarkable minerals [1]. General undersea engineering comprises of an extensive variety of sensors placed in oceanic surfaces to collect data inside a particular circumstance. In underwater environment the sensor nodes are normally considered as static yet it is additionally viewed as that it might move from 1 to 3 meters for each second as a result of the water stream [2].The sensors are dynamic and move as per the sea currents. Underwater is frequently viewed as an unforgiving domain where sensors tend to move from a particular point to an unexpected area [3]. The restriction of underwater circumstance is mainly because of battery source for sensors added to the fact that it is difficult to the batteries in the deep ocean. Static sink additionally makes hotspot issues where the nearest sensors to the sink demise at an early phase riding off few zones ungoverned. It can be relieved by portable or movable sinks in deep waters. Connectivity between nodes is also an issue because of void regions. For underwater applications multipath or multihop networks are necessary for passing the data and forwarding it to the sink. Once the sink node receives the data it forward to the concerned node through radio communication. The design of routing protocol faces problem even because of localization issues, This research papers focuses on the mobility models for routing which supports water pressure and water current[4].

\subsection{Major Challenges in Underwater}

Designing of a reliable protocol is one of the challenging areas in underwater environment. However, the designing of such protocol faces some challenging issues.
- The Underwater environment is severely affected by limited available bandwidth.

- Propagation Delay in water is 5 times greater than that of Radio frequency.

- High Bit Rate and temporary losses of connectivity arise due to extreme water condition.

- Multipath fading makes the channel to be severely impaired.

- Limited power as battery cannot be replaced or recharged.

- Due to the self motion capability of the water current there is change in the network topology.

- Difficulty in routing as there is a high movement of nodes in water current.

- Subjected to failure because of fouling and corrosion.

\subsection{Architecture of UWSN}

The architecture of underwater wireless sensor node is shown in Figure 1.The power unit is responsible to manage the energy consumption of the nodes [5].

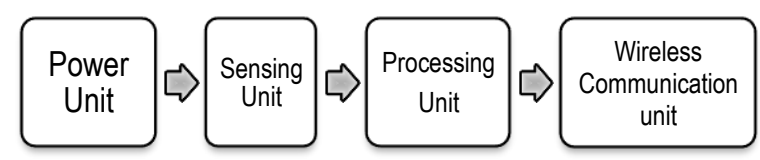

Fig.1: Architecture for UWSN

The Sensing unit is utilized to get information .It is dynamic all the time, even when the node is in rest [6]. The Processing unit is obligated to process all sort of information. The wireless communication unit is in charge for communicating the data [7]. 


\section{Routing Protocols Based on Node Mobility}

The design of a reliable and efficient routing protocols based on node mobility for underwater condition is a issue of demand. So there is a requirement to design efficient routing algorithms for mobile nodes. Here in this section the deployment and arrangements of nodes, its construction, route analysis, route maintaining, and packet advancing schemes are discussed. Figure 2 describes the classification of routing algorithm related to mobile nodes.

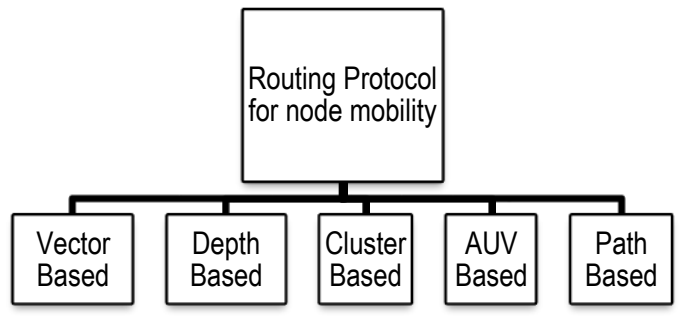

Fig. 2: Node Mobility based routing Protocols.

\subsection{Vector Based Protocol}

In sensor networks, power consumption is always a major problem as it keeps running the battery, so it is challenging to recharge them in many rejuvenate situations. In undersea sensor networks, apart from energy preserving, the routing method should be capable to compromise with mobility of a node in a qualified manner.

VBF is basically uses a location-based routing methodology. There is no need of state information on the sensor nodes but only a few fractions of the nodes are employed in routing. However, packets involved will be moving in superfluous and interleaved direction from source to goal, which give robustness, scalability and is also energy efficient to VBF. VBF is basically a combination of localization algorithm and routing algorithm in which the localization and routing are performed together. By incorporating such interleaved and redundant way forwarding packets it is strongly secured against node failure and packet loss. In addition VBF is also basically a geographic routing algorithm. In $\mathrm{VBF}$, every packet conveys the places of the sender, the destination and the forwarding node which transmits the data. The sending pathway is indicated by the directing vector to the objective from sender. After acquiring a data, each node finds its proportionate place to the advancing node by estimating its separation to the advancer node and the angle of arrival (AOA) of the signal. Repeatedly, every one of the nodes acquiring the packets processes its positions. In the event if it is confirms that a node is near to the routing vector i.e., less than a pre calculated stretch limit, it adds its processed position in the data and continues sending data packets; or else, it will just drop the data packet.

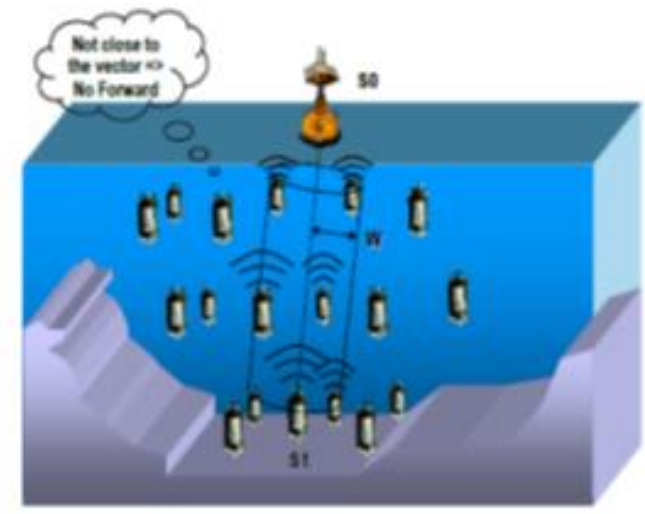

Fig. 3: VBF for UWSNs
In the figure 3 the fundamental idea of VBF is visualized, node S1 is the source, and node S0 is the sink. Sender form a routing channel along the routing vector and with a pre-defined radius indicated by $\mathrm{W}$. The positioning of nodes is allotted locally and no global wide synchronization needed.VBF has no prerequisite for stable forward way. The accomplishment of data packet delivery is not subject to steady neighbourhood, but depends on the denseness. If the event there exists no less than one way in the "routing channel pipe" determined by the routing vector, then the data packet can be effectively transported. The calculation demand on every node is proper for routing on-interest since just straight forward calculation is required. VBF is strongly secure against missing packets and collapse nodes. VBF is robust towards node failure since there is no $\mathrm{ACK}$ or retransmission for a lost packet in the protocol.

\subsubsection{Hop by Hop Vector-Based Forwarding Protocol.}

HHVBF protocol routing is flooding approach. H2VBF is developed after $\mathrm{VBF}$ protocol [8], is a notable routing protocol designed for undersea sensor networks. In VBF, the flooding approach is invoked taking the separation between the participating nodes is little than the virtual routing channel. The distinct difference of HHVBF over VBF is that ,in VBF from source to destination a single routing vector is assumed, but, in $\mathrm{HH}-\mathrm{VBF}$ from each sender to the target the routing vector is performed.

After receiving a data, the node calculates the vector from starting of node to the sink node. If the range or stretch between the calculated vector and node itself is small over the radius of the virtual routing channel, then the node becomes qualified for sending and turns into a competitor advancer .The sending node which is eligible for forwarding waits for a particular time before it is transmitted is called holding time.

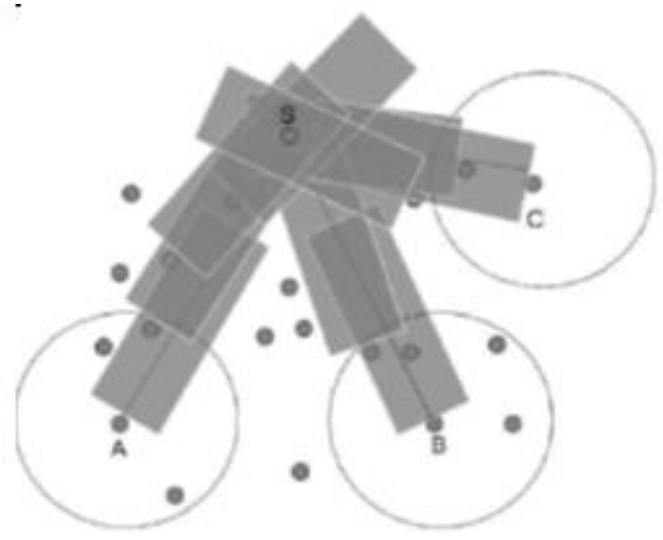

Fig. 4: Hop by Hop Vector-Based Forwarding scenario

Figure 4 shows the HH-VBF scheme, in the figure $\mathrm{S}$ is the sink node or the destination node and A, B, C are the source nodes [9]. The rectangle represents the zone covered by the virtual channel or pipe of every node. Here in HH-VBF the copy (duplicate) of the same data packet from its neighbour is eliminated when it is ready for transmitting the packets from source to sink. It avoids duplicating the packet by calculating the distance from each node and the vector of the forwarding node. If the calculated distance is larger than the defined threshold, the packets are further transmitted or else the packet is dropped.

\subsubsection{Energy-Saving Vector Based Forwarding Protocol.}

The ESVBF concentrates on the information of nodes energy which sends data packet continuously within the steering pipe [8]. ESVBF produces the preferable outcomes than VBF.The problem with ESVBF is that, it is not suitable for removal of voids which would ultimately drop the data packets and result in poor delivery ratio of packet transmission [10]. The routing algorithm ESVBF is 
best suitable for the nodes that are within the limit of the steering pipe; if the node stay away from the routing channel it may discard the data packets and would be depleted soon and hence decrease the power level.

\subsubsection{Cluster Vector Based Forwarding (CVBF) Protocol}

Cluster Vector Based forwarding method takes thick zone of undersea to improve the data packet distribution time and to minimise the end to end delay. CVBF is considered to be superior to all other protocol. CVBF method partitions the entire network into many qualified clusters [11].In each cluster group, the associate nodes are choosen with respective to their geographic position. One node at the highest point of each group is considered as virtual sink node. The remaining node in the group sends information packets to their corresponding sink in the cluster.

CVBF characterizes on virtual routing channel or pipe for each and every cluster. The radius of the routing channel is equivalent to the communication bound of the node. When sink node in each cluster group starts receiving the packet from the source sensor, it will collect all the data and then transmit to the main sink node, which is on the shallow surface of water. It is done through one hop method. But CVBF approach is not that easy when multiple cluster and multiple routing channel is taken in to consideration for under water scenario, due to water current, because of which packets may drop and so affect the delivery ratio of data.

\subsection{Depth-Based Routing (DBR) Protocol.}

Depth based routing protocol uses the depth information of the sensor node. The details regarding of the depth of the node is obtained from the sensor connected to the node. The DBR protocol tells the necessity of localization. In DBR every node adds its depth details to the data packet. The node, after accepting the data it checks for the receiving nodes depth. If the obtained depth information is smaller than the transmitter depth, the node will transmit the packet, this depth based routing protocol does not require the entire location information.Rather, it needs only the depth information [12].

The advantage of the protocol is that it can deal with dynamic structure of the network effectively without the help of a localization services. The detail of the depth information is updated at each and every hop of the forwarder node.

The goal of the DBR is sending packet when it has smaller depth or else it may reject the packet. DBR has two main queues in order to minimise number of sending nodes. The first is the priority based queue and the later is the buffer based queue.

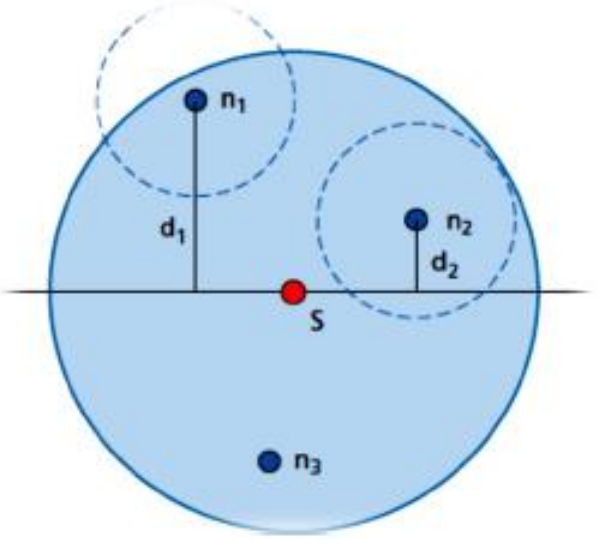

Fig. 5: Data Transmission in DBR

DBR keeps the repetition of the packets in control, this is done by checking the buffer periodically. The scheduling time in DBR depends on the priority based queue of the packets and waiting time depends on the arrival of the packets and hop of the previous packet. Though it do not need the complete information of the zone information and can deal with the network dynamically .It ends up with high transmission delay, huge packet colliding and more battery consumption, this is all due the multiple duplication of data packets.

Figure 5 describes the data transmission scenario of DBR.The sender node is given by $\mathrm{S}$ and the neighbours are named as $\mathrm{n} 1, \mathrm{n} 2$, $\mathrm{n} 3$ respectively. The sending node say $\mathrm{n} 1$ closer to main sink at the receiving end than the $\mathrm{n} 2$ node. Node $\mathrm{n} 1$ would transmit the data packets as it is close to the destination, node 2 will be in the wait mode. The packets remain till the waiting period, after which it transmits the data and also intimates to the buffer about the transmission so that it can take control over duplication. The Transmission of data packets here is based on the details of depth of the current node and the previous node.

\subsection{1 $\mathrm{H}^{2} \mathrm{DAB}$}

Hop by Hop Dynamic Addressing Based Protocol is also based on flooding method along with a unique identity of sensor nodes.H2DAB assigns a special unique identity [13] to each node may be unique ID or hop ID, which gives you the separation between the sink node and the sender node. H2DAB divides the water into different levels, the nodes are placed in bottom level and few nodes are placed in the other water levels. The movement of the node is controlled by selecting the address dynamically. Addressing is done from small depth level to large depth level. Small address is given to the destination nodes and large address is given to the sender nodes. Small address are given to the node which is closer to the sink node, large address are given to nodes at the bottom level.

Hello packets are used to the one which generates the dynamic addressing.H2DAB gives high delivery ratio, because of unique identity for hop and the selected nodes move horizontally.H2DAB also takes parameter from dense network.

\subsubsection{Delay-Sensitive Depth Based Routing.}

The DSDBR is the enhanced adaptation of DBR. DSDBR depends on holding time profundity data, and profundity threshold [14]. The sensor node in DSDBR is detected and the transmitted information is inside the communication scope. The packets will be transmitted by source node to those neighbours which brings down profundity. At the point when neighbour will get the information bundles it will figure the holding time and profundity limit. Profundity edge restrains the profundity of present and past hub amid exchange of data. Depth limit is relies on weight capacity and weight capacity can be ascertained over system measure and qualified neighbour sending esteems. DSDBR technique isn't appropriate for expulsion of void districts. In the event that any nodes in the scope of void districts the particular node will shed the data and in turn brings down the distribution ratio

\subsubsection{Delay-Sensitive Energy Efficient Depth Based Routing (DSEEDBR}

The DSEEDBR enhances system lifetime along with delay responsively in EEDBR with depth information [14]. In DSEEDBR the advancing nodes figures the failure in transmission, channel noise and difference of intensity with time slack of the node to be sent in order to solve the energy issue. But calculation of depth information of the sender nodes makes it too burden and will pass on soon, so claim of essentialness adequacy for DSEEDBR isn't trustworthy.

\subsection{Clustered Based Routing Protocols}

Clusters are formed depending on the movement of the nodes. In each cluster the cluster head and the members of the group are assigned. Cluster head is mainly responsible for communication between the group and the main sink node on the surface. It 
receives data from the members in the group and advancing the data to the destination sink node.

\subsubsection{Hydraulic Pressure Based Any Cast Routing (HydroCast)}

HydroCast is relays on cluster routing. It is actually a geographically spreader localization routing [15].It transmits the data to the sink node by measuring the pressure levels. As in DBR, this protocol used the depth information with the respective cluster with no hidden information of source node, and it is done through pressure levels. The maximum throughput is obtained from the clusters near to the sink.

In HydroCast engineering the clusters are shaped with the most extreme dynamic of those nodes which are nearer to the goal node. The greatest dynamic can be ascertained through cluster conveyance and likelihood criterion. A sensor node which is the piece of the cluster, the data of that node will be installed in the data packet design format. In information sending component the greatest dynamic node has most elevated need. The greatest advance node has most limited timeout for communication. In HydroCast convention the nearby most extreme restoration technique is utilized, which plays out the constrained flooding component approach. In the flooding method the neighbourhood most extreme node is known as the performer node. The tetra level technique has likewise been utilized as a part of the planning of this routing; this strategy will recognize the neighbour node for nearby most extreme node. The nearby greatest node exchanges the data to other neighbourhood most extreme nodes through predetermined number of jumps to the sink. HydroCast has utilized the eager based system for evacuating of void locales which upgrades the information delivery rate. In HydroCast the different quantities of same packets are gotten by sink node which upgrades the additional heap on network.

\subsection{AUV Based Routing Protocols}

Routing algorithm which relays on Autonomous Underwater Vehicles (AUVs) mobility are called the AUV based routing protocols. Mobicast routing protocol depicted with the mobility nodes.

\subsubsection{Mobicast}

Mobicast routing algorithm is found energy efficient for UWSN, it resolves the issue of power management and capricious 3D hole. The power utilization of nodes is profoundly low in Mobicast[16]. Creating user defined routing in 3D environment is difficult due to water current. Data delivery proportion is high, the protocol diminishes the utilization of power because of sensor nodes and the data overheads. The protocol upgrades the information conveyance proportion. Because of nonstop water development, if the dynamic nodes may stay far from AUV, then the dynamic nodes can drop the packets and at last resulting in low delivery ratio.

\subsection{Path Based Routing Protocol}

Path based routing protocols builds up the single or numerous way from source to sink nodes. This different way method enhances the packet delivery ratio in contrast with single way.

\subsubsection{Multi-layer Routing Protocol.}

MRP coordinating tradition is used to decide the issue of confinement and updates the battery life of standard sensor node[17]. The framework plan of MRP relies upon sink centers, super center points and sensor center points. The sink center points are determined to the water surface and super center points are settled and spread in different water levels. Sensor center points are sent at the base of the water. MRP develops the 2D layers around the super center point. MRP is used to develop the layer ID and sensor center point ID for packages sending with different power levels as shown in Figure 6.

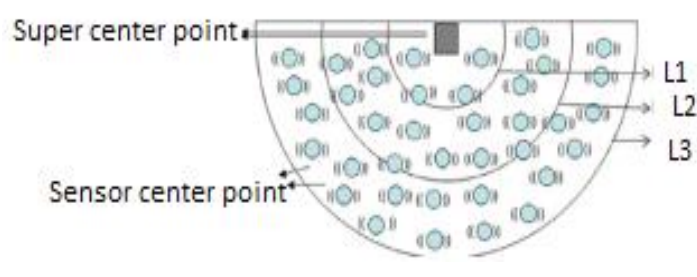

Fig. 6: Different power levels

The super center point is used to redesign the battery life of typical sensor centers. MRP uses the 2D center point association segment however submerged support 3D course of action. In MRP the packages holding time computation isn't genuinely described. If sensor center point may avoid super center than the sensor center may drop the packages and finally the groups movement extent might be impacted.

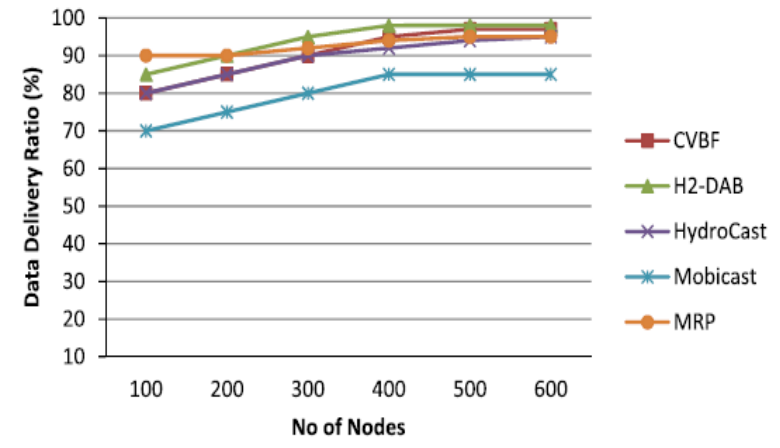

\section{Conclusion}

Routing for mobile USWN is one of the testing issues. Over the previous years large numbers of the routing protocols based on node mobility have been proposed with various attributes. Each routing protocol is discussed with mobility of nodes, data transferring, route creation, route maintenance, and performance issues. It is watched that the execution $\mathrm{H} 2-\mathrm{DAB}$ is superior to any other protocol because the depth addressing mechanism of $\mathrm{H} 2$ $\mathrm{DAB}$ covers the node movement in proficient way

\section{References}

[1] Li, N., et al., 2016. A survey on Underwater Acoustic Sensor Network Routing Protocols. Sensors 16 (3), 414.

[2] E. Felemban, F. K. Shaikh, U. M. Qureshi, A. A. Sheikh, and S. B.Qaisar, "Underwater sensor network applications: a comprehensive survey," International Journal of Distributed Sensor Networks, vol. 2015, Article ID 896832, 2015.

[3] Azam, I., et al. SEEC: Sparsity-aware energy efficient clustering protocol for underwater wireless sensor networks. in 2016 IEEE 30th International Conference on Advanced Information Networking and Applications (AINA). 2016. IEEE.

[4] Chen, K., Zhou, Y., He, J., 2009. A localization scheme for underwater wireless sensor networks. Int. J. Adv. Sci. Technol. 4

[5] J. L. Tangorra, S. N. Davidson, I. W. Hunter et al., "The development of a biologically inspired propulsor for unmanned underwater vehicles," IEEE Journal of Oceanic Engineering, vol.32, no. 3, pp. 533-550, 2007

[6] W. K.G. Seah andH. P. Tan, "Multipath virtual sink architecture for wireless sensor networks in harsh environments," in Proceedings of 1st International Conference on Integrated Internet Adhoc and Sensor Networks, fra, May 2006.

[7] R. Manjula and S. S. Manvi, "Issues in underwater acoustic sensor networks," in Proceedings of, vol. 3, p. 101, 2011. 
[8] P Xie, JH Cui, L Lao, "VBF: vector-based forwarding protocol for Underwater Sensor Networks", IFIP NETWORKING, pp. 1216-1221, 2006.

[9] Nicolas Nicolaou, Andrew See, Peng Xie, "Improving the robustness of location-based routing for Underwater Sensor networks", IEEE OCEANS 2007, pp. 1-6, 2007.

[10] Wei, B.. et al. 2012. ES-VBF: an energy saving routing protocol. In: Proceedings of the International Conference on Information Technology and Software Engineering. 2013. Springer.

[11] Ibrahim, D.M., et al. Enhancing the vector-based forwarding routing protocol for Underwater Wireless Sensor Networks: a clustering approach. In: Proceedings of the Tenth International Conference on Wireless and Mobile Communications (ICWMC), 2014.

[12] Hai Yan, Zhijie Shi, Jun-Hong Cui, "DBR: Depth based Routing for Underwater Sensor Networks", Springerlink NETWORKING, pp.16-1221, 2008.

[13] Ayaz M, Azween Abdullah, "Hop-by-HopnHop Dynamic Addressing Based (H2-DAB) Routing Protocol for Underwater Wireless Sensor Networks", IEEE international conference on Information and Multimedia Technology ICIMT '09, pp. 436441, 2009.

[14] Javaid, N., et al., 2015. Delay-sensitive routing schemes for Underwater Acoustic Sensor Networks. Int. J. Distrib. Sens. Netw. 2015.

[15] Lee, U.. et al. 2010. Pressure routing for underwater sensor networks. In: Proceedings of IEEE INFOCOM.

[16] Chen, Y.-S., Lin, Y.-W., 2013. Mobicast routing protocol for underwater sensor networks. Sens. J. IEEE 13 (2), 737-749.

[17] Wahid, A., et al., 2014. MRP: a localization-free multi-layered routing protocol for Underwater Wireless Sensor Networks. Wirel. Pers. Commun. 77 (4), 2997-3012

[18] K Mahalakshmi, D.R Prabhakar, D.V Balakrishnan, "Kernel optimization for Improved Non functional requirement classification" Journal of Theoretical and Applied Information Technology 60(1), 64-72.

[19] K. Mahalakshmi, R Prabhakar "Performance Evaluation for Nonfunctional Requirements" Global Journal of Computer Science and Technology 13 (8).

[20] K Mahalakshmi, R Prabhakar, V Balakrishnan, “ Optimizing Support Vector machine" for classifying non functional Requirements" Research Journal of Applied Sciences, Engineering and Technology 7 (17).

[21] K. Mahalakshmi, "Hybrid optimization of SVM for improved Nonfunctional requirement classification" International Journal of Applied Engineering Research 10 (20), 20157-20174.

[22] K Mahalakshmi, S Manikandan, S Nithyanantham, "ENHANCED SOFTWARE ENGINEERING PROCESS MODEL FOR GREEN ENVIRONMENT IN VIEW OF ACHIEVING SUSTAINABILITY" , International Journal of Advanced Engineering Technology, Vol 7/2, June 2016, 1072 1079

[23] K.Shanthakumar, K.Mahalakshmi, R. Keerthika, "AUGMENTED PERSPECTIVE MINING METHODOLOGY FOR CONTROLLED SOCIAL MEDIA", Pakistan Journal of Biotechnology, 2017/12, V14, 340-343

[24] K.Mahalakshmi, "Dynamic Virtual Machines Building Utilizing KBR Agent For Information Security In Hybrid Cloud", Journal of Advanced Research in Dynamical and Control Systems (JARDCS), 2017/06, 1181 - 1190, Institute of Advanced Scientific Research

[25] S.V. Manikanthan , T. Padmapriya "An enhanced distributed evolved node-b architecture in 5G tele-communications network" International Journal of Engineering \& Technology (UAE), Vol 7 Issues No (2.8) (2018) 248-254.March2018

[26] S.V.Manikanthan and T.Padmapriya "Recent Trends In M2m Communications In 4g Networks And Evolution Towards 5g", International Journal of Pure and Applied Mathematics, ISSN NO:1314-3395, Vol-115, Issue -8, Sep 2017.

[27] S.V.Manikanthan, Padmapriya.T, "RECENT TRENDS IN M2M COMMUNICATIONS IN 4G NETWORKS AND EVOLUTION TOWARDS 5G", International Journal of Pure and Applied Mathematics, Vol. 115, No. 8, pp: 623-630, 2017. 